\title{
Impact of COVID-19 lockdown on suicide attempts
}

\author{
A retrospective analysis of the springtime admissions to the trauma \\ resuscitation room at the Medical University of Vienna from 2015-2020
}

\author{
Greta L. Carlin (D) · Josef S. Baumgartner (D) · Timon Moftakhar · Daniel König (D) • Lukas L. Negrin (DD
}

Received: 16 September 2020 / Accepted: 20 February 2021 / Published online: 31 March 2021

(C) The Author(s) 2021

\begin{abstract}
Summary
Background In response to the current coronavirus disease 2019 (COVID-19) pandemic the Austrian government issued a lockdown from 16 March to 15 May 2020. As periods of economic and emotional burden have proven to detrimentally affect people's psychological health, healthcare officials warned that the strict measures could have a serious impact on psychological health, leading to an increase in suicide attempts. Thus, the objective was to provide evidence for this assumption.

Methods All adult patients admitted to the trauma resuscitation room of the Medical University of Vienna during the lockdown period following a suicide attempt were included in this analysis, forming the study group. Suicidal patients treated during the same period in 2015, 2016, 2018, and 2019 were pooled to create the control group. The year 2017 was excluded because another major hospital in Vienna was partially closed due to a change in infrastructure, resulting in an increased number of severely injured patients treated at our department. As the lockdown caused a markedly decreased number of patients admitted due to other reasons than suicide the actual number was replaced with the average number of patients admitted in the relevant previous 4 years.
\end{abstract}

Greta S. Carlin and Josef S. Baumgartner are shared first authors.

G. L. Carlin $(\bowtie) \cdot$ T. Moftakhar $\cdot$ L. L. Negrin

Department of Orthopaedics and Trauma-Surgery, Medical University of Vienna, Vienna, Austria

greta.carlin@meduniwien.ac.at

J. S. Baumgartner · D. König

Clinical Division of Social Psychiatry, Department of

Psychiatry and Psychotherapy, Medical University of Vienna,

Vienna, Austria
Results By comparing the study and the control group based on this realistic estimate we revealed an increase in attempted suicides during the lockdown period ( $p=0.001)$. Demographic, mental health-related, and injury specific patient data did not differ between the groups.

Conclusion The results strongly urge for an improvement in crisis intervention and suicide prevention measures in the event of a future lockdown.

Keywords COVID-19 pandemic $\cdot$ Suicide attempts during lockdown · Coronavirus lockdown . Polytrauma $\cdot$ Suicide prevention

\section{Introduction}

Suicide is the second leading cause of traumatic death worldwide, surpassed only by road traffic collisions [1]. The World Health Organization (WHO) estimated that in 2016 around 793,000 people died by suicide and while the global total number seems to be decreasing, various countries such as the USA, South Korea and Japan are reporting rising trends [2-6]. In 2018, 1209 people died from suicide in Austria, the most common methods were hanging (45\%), shooting (19\%), jumping from a height and poisoning (10\% each), jumping in front of a moving object (7\%), drowning (3\%), cutting, burning or causing an accident [7].

Suicide attempts are a serious, potentially preventable healthcare problem. On the one hand, trying to commit suicide may be a cry for help, a conscious or unconscious method for getting others to recognize just how badly the individual is feeling. On the other hand, contemplating suicide may be caused by a very pronounced death wish. Considering the mechanisms of injury and the physics behind them, it seems clear that the first group of individuals attempting suicide 
preferably choose methods, such as cutting, which mostly injure only one body region (monotrauma), not immediately leading to death and allowing the injured to call for help in case they change their mind; however, individuals, who are determined to commit suicide, preferably select irrevocable methods that result in multiple injuries (polytrauma), unlikely to survive, such as jumping from great heights or jumping in front of a train. Moreover, polytrauma patients pose a unique challenge to the team treating them, frequently requiring a multidisciplinary approach with the individual outcome remaining hard to predict [8], resulting in a significant socioeconomic burden. By investigating the changes in the number of patients admitted to the trauma resuscitation room of the Medical University of Vienna following intentionally caused trauma (differentiating between polytrauma and nonpolytrauma) we were able to evince the effect of the lockdown on people's psychological health.

The current COVID-19 pandemic has spread rapidly around the globe and become an international health concern [9]. Furthermore, it also negatively impacts the economic stability of various countries and thus directly affects the financial livelihood of many people [10]. In order to flatten the hospitalization curve and thus not overload the healthcare system, the Austrian government issued a set of strict measures and social restrictions beginning on 16 March 2020. All non-essential businesses were closed, and most workplaces established home office working. Kindergartens, schools and universities were shut for the foreseeable future. Especially senior citizens were asked to remain at home given their increased vulnerability [11]. These measures lasted up to 15 May 2020, when the lockdown officially ended. Similar measures were introduced in many other countries. Accordingly, the possibility to get in touch with medical caregivers was reduced as ambulatory healthcare for non-emergency patients could not be provided as extensively as usual. Additionally, individual patient evaluation as well as various treatment options, such as elective surgery were drastically affected by COVID19 [12-17]. Furthermore, by social distancing from friends and family, individuals appeared to be completely left alone with often compromising afflictions, hence causing alarming psychological distress [18].

From the start, healthcare providers worldwide warned that the lockdown for the current COVID-19 pandemic could result in unemployment and lead to increased anxiety and depression $[10,19,20]$. In addition, social distancing measures could confluence to increase known risk factors for suicidal behavior, such as living alone, loneliness and social isolation $[21,22]$. The possibility of an increasing occurrence of suicide attempts, especially in more vulnerable populations, such as senior citizens and those already suffering from mental illness was further discussed [23-26]. Until now there are no data on the effects of the COVID-19 crisis on suicide attempts.
The aim of the present study was to analyze how many of the patients admitted to the trauma resuscitation room of the level 1 Trauma Centre of the Medical University of Vienna during the COVID-19 lockdown in Austria were injured due to a suicide attempt by intentionally caused trauma. Secondly, we investigated how many of those were classified as a polytrauma and whether these numbers or any of the demographic, socioeconomic and health-specific variables collected differed from the patients admitted following a suicide attempt during the same time period in the previous 5 years. The authors hoped to thereby gain more insight for healthcare professionals should a future lockdown or pandemic occur.

\section{Methods}

\section{Data source}

Our retrospective data analysis, which was approved by the local ethics committee (EK no: 1607/2020), focused on all patients (1) with a minimum age of 18 years, (2) who were admitted to the level 1 Trauma Centre of the Medical University of Vienna during the lockdown period established by the Austrian government (16 March-15 May 2020), and (3) for whom treatment in the trauma resuscitation room (shock room) was considered appropriate due to the injury mechanism. These patients were subdivided into trauma victims and individuals who had attempted suicide. Of the latter, demographic data including age, gender, outcome (survival/fatality), ethnic minority background, known substance abuse, known psychiatric diagnosis, previous suicide attempt, previous psychiatric treatment, abbreviated injury scale (AIS) for the regions head or neck $\left(\mathrm{AIS}_{\mathrm{Head}}\right)$, face $\left(\mathrm{AIS}_{\mathrm{Face}}\right)$, thorax (AIS Thorax ), abdominal or pelvic contents (AIS Abdomen $_{\text {), }}$ extremities or pelvic girdle (AIS Extremities$_{\text {), and external }}$

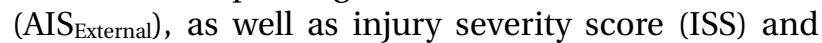
zip code of residence district were extracted from patients' medical charts. Of our study group, a special focus was set on polytrauma victims, defined by an ISS $\geq 16$. In order to be able to form a valid control group all patients who were admitted to the level 1 Trauma Centre of the Medical University of Vienna following a suicide attempt from 16 March to 15 May in the years 2015-2019, were assessed. In general, survival referred to the period of hospitalization.

\section{Statistics}

All patient records were pseudoanonymized and deidentified prior to statistical analysis that was performed using the SPSS system (IBM, Armonk, NY, USA, version 26). Normally distributed variables are presented as mean \pm standard deviation, non-normally distributed variables and categorical data are characterized by median and range in square brackets, and dichotomous characteristics are displayed 
by absolute and relative frequencies. The MannWhitney U-test was applied to compare continuous variables, whereas categorical data were analysed by means of the $\chi^{2}$-test. In general, a $p$-value $<0.05$ was considered significant.

\section{Results}

Our database search provided the numbers presented in Table 1. Fig. 1 displays the absolute frequencies of accidental and intentionally caused trauma depending on the year of admission, revealing a different distribution $(p<0.001)$. Fig. 2 shows the proportion of polytrauma victims based on all patients who had attempted suicide, which did not differ significantly across the years 2015-2020 $(p=0.102)$.

Since we detected a spike of suicide-associated polytrauma patients admitted to our institution for the 2-month period in 2017 we further investigated the reason for this occurrence, revealing that data were not comparable (as specified in the discussion) and thus had to be excluded from our statistical analysis. In consequence, our control group was formed by all patients admitted to the trauma resuscitation
Fig. 1 Patients admitted to resuscitation room (suicidal vs. non-suicidal) in the same 2-month period from 2015 to 2020

Fig. 2 Patients after suicide attempt (polytrauma vs. non-polytrauma) in the same 2-month period from 2015 to 2020
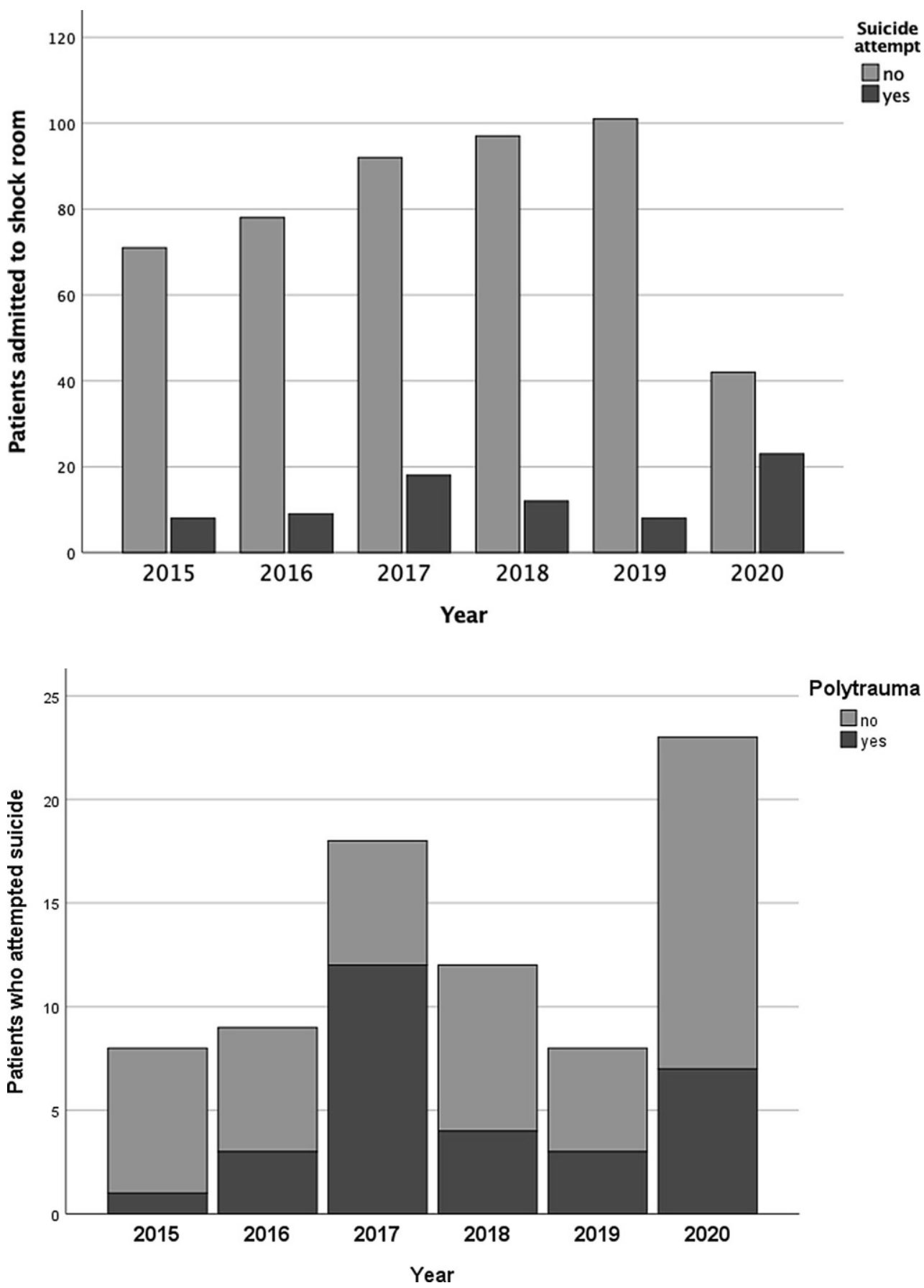
Table 1 Number of patients admitted to the trauma resuscitation room according to years

\begin{tabular}{ll|l|l|l|l|l|} 
Number & \multicolumn{7}{l}{ Year } & & & & & \\
\cline { 2 - 7 } & 2015 & 2016 & 2017 & 2018 & 2019 & 2020 \\
\hline Admissions & 79 & 87 & 110 & 109 & 109 & 65 \\
\hline $\begin{array}{l}\text { Attempted sui- } \\
\text { cides }\end{array}$ & 8 & 9 & 18 & 12 & 8 & 23 \\
& $(10.1 \%)$ & $(10.3 \%)$ & $(16.4 \%)$ & $(11.0 \%)$ & $(7.3 \%)$ & $(35.4 \%)$ \\
\hline Polytrauma & 1 & 3 & 12 & 4 & 3 & 7 \\
& $(1.3 \%)$ & $(3.4 \%)$ & $(10.9 \%)$ & $(3.7 \%)$ & $(2.8 \%)$ & $(10.8 \%)$ \\
& & & & & &
\end{tabular}

Table 2 Demographic, injury-specific and mental healthrelated data of the patients in the control and in the study group

\begin{tabular}{|l|l|l|}
\hline & Control group & Study group \\
\hline Number of patients & 37 & 23 \\
\hline Deceased & 2 & 1 \\
\hline Age (years) & $(5.4 \%)$ & $(4.3 \%)$ \\
\hline Male:female & $43.2 \pm 17.6$ & $38.7 \pm 12.2$ \\
\hline ISS & $29: 8$ & $17: 6$ \\
\hline Polytrauma & 4 & 4 \\
\hline Known substance abuse & {$[1-75]$} & {$[1-43]$} \\
\hline Known psychiatric disease & 11 & 7 \\
\hline Previous suicide attempt & $(29.7 \%)$ & $(30.4 \%)$ \\
\hline Previous psychiatric treatment & 18 & 15 \\
\hline Minority background & $(48.6 \%)$ & $(65.2 \%)$ \\
\hline Suicide method used & 32 & 19 \\
\hline Jump from a height & $(86.5 \%)$ & $(82.6 \%)$ \\
\hline Jump in front of a moving object & 13 & 9 \\
\hline Cutting & $(35.1 \%)$ & $(39.1 \%)$ \\
\hline Driving off the street & 25 & 12 \\
\hline Self-immolation & $(67.6 \%)$ & $(52.2 \%)$ \\
\hline Hanging & 10 & 9 \\
\hline Shooting & $(27.0 \%)$ & $(39.1 \%)$ \\
\hline etc. & & \\
\hline ISS Injury Severity Score & 9 & 10 \\
\hline & $(24.3 \%)$ & $(43.5 \%)$ \\
\hline & $16.5 \%)$ & 2 \\
$(8.7 \%)$ \\
\hline
\end{tabular}

room in 2015, 2016, 2018, and 2019 following a suicide attempt, whereas all suicidal patients treated in 2020 were combined to our study group. As an effect of the lockdown the number of patients admitted due to other causes than suicide decreased markedly in
2020, necessitating a realistic estimate of this number to shed light on the research question regarding a potential increase in suicides as hypothesized. We decided to replace the actual number of patients admitted for other reasons than suicide in 2020 with 87 , the average number of patients admitted in 2015 , 2016, 2018, and 2019, resulting in a total of 110 fictive admissions to the trauma resuscitation room in 2020 , including $20.9 \%$ attempted suicides and $6.4 \%$ suicidal polytraumas.

Based on this estimate we revealed a significantly higher proportion of attempted suicides in all patients admitted to the trauma resuscitation room $(p=0.001)$ during the lockdown period in 2020 compared to the same 2 -month time period in the pooled previous 4 years, whereas no difference in the proportion of suicidal polytrauma could be found ( $p=0.084$ ).

In order to detect any differences in demographic, injury-specific and mental health-related data, we compared the patients in the study group with those in the control group. The results are presented in Table 2. As expected, subgroup analyses did not reveal significant differences due to the small number of patients $(p \geq 0.210)$. Moreover, significant differences in injury severity, assessed by the AIS, could not be calculated $(p \geq 0.086)$.

Of the 23 patients in our study group, 7 sustained polytrauma (age 35.1 \pm 13.5 years; ISS, 41 [22-43]), 5 males jumped from a height, 1 female jumped in front of a train (she was the only patient who died) and 1 female performed self-immolation. The control group included 11 individuals suffering from polytrauma

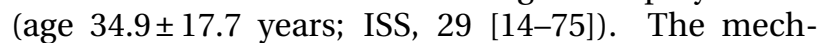
anisms of injury were jumping from a height (4), jumping in front of a moving object (4), cutting (1) and driving off the street (2).

In the study group the subdivision of the patients in those sustaining/not sustaining polytrauma according to the week of admission during lockdown is presented in Fig. 3, not reaching statistical significance $(p=0.577)$.

Finally, no cluster regarding shared areas of residence could be detected for individuals admitted due to suicide attempts.

\section{Discussion}

\section{Main findings}

During the national COVID-19 lockdown in Austria we identified a higher percentage of suicide-associated admissions to the resuscitation room of our level 1 Trauma Centre compared to the same 2-month time period in each of the previous 5 years. These differences did not necessarily indicate that there were more suicide attempts in 2020 as the number of patients admitted due to other reasons than suicide was lower than average. Moreover, it turned out that the year 2017 was not comparable to the other years 
Fig. 3 Patients after suicide attempt (polytrauma vs. non-polytrauma) in each week of the lockdown

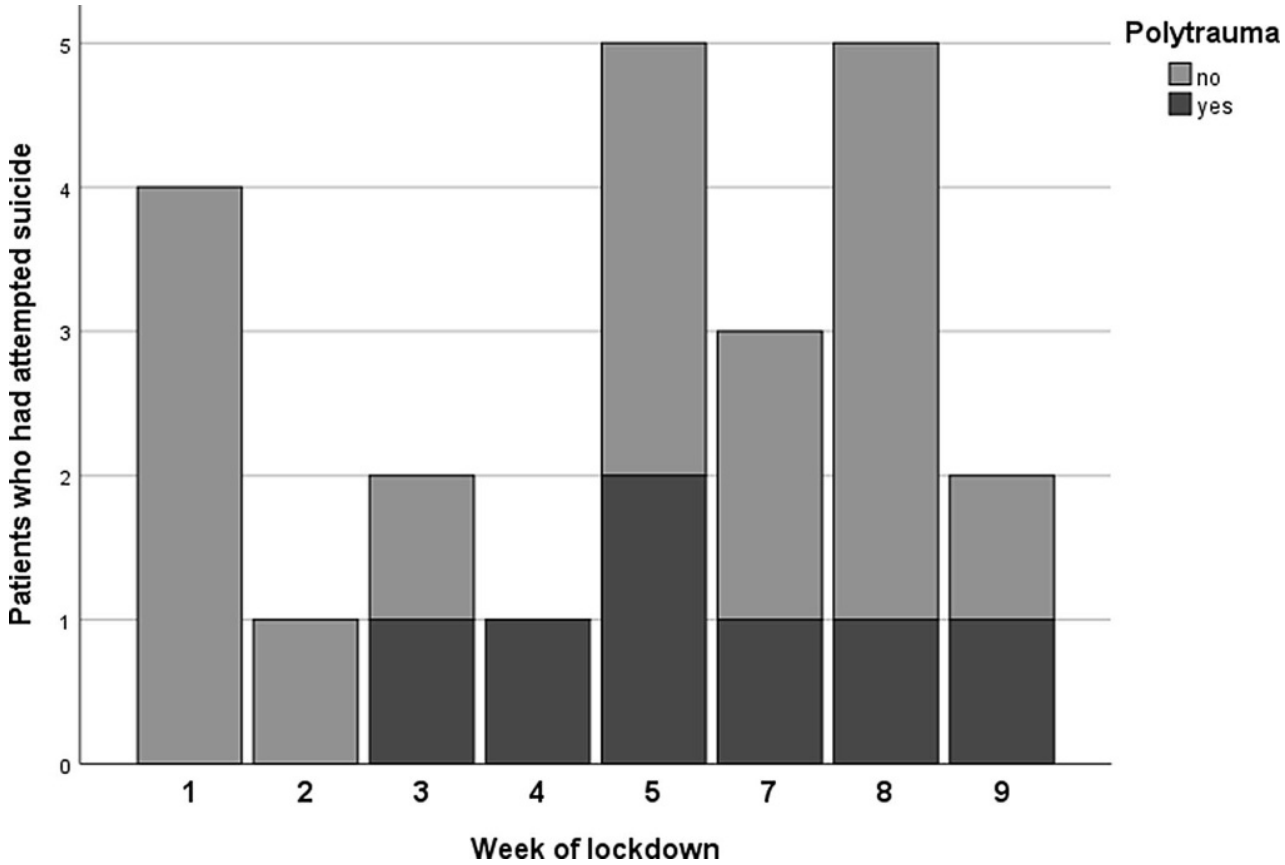

2015-2019, necessitating exclusion of the data for 2017 from our analysis. Since the number of patients admitted to the trauma resuscitation room due to reasons other than suicide markedly decreased during the lockdown, we replaced this number by the mean number of patients admitted in 2015, 2016, 2018, and 2019 due to reasons other than suicide. Even after this adaptation a higher percentage of attempted suicides $(p=0.001)$ was revealed in the study group compared to the control group; however, a higher percentage of patients suffering an intentionally caused polytrauma could not be revealed ( $p=0.084)$.

Regarding the mechanism of injury, most attempted suicides were performed by jumping from a height during the COVID-19 lockdown, whereas the majority of patients in the control group had presented after trying to kill themselves through cutting. Finally, the ratio of males and females, age and overall injury severity as well as known risk factors for suicide, such as ethnic minority status, socioeconomic background, substance abuse, pre-existing psychiatric diagnosis, previous suicide attempt and previous psychiatric treatment, did not differ significantly between the study and the control group.

\section{Interpretation of the findings}

As the lockdown measures took effect and people were advised to "stay at home" we noticed a reduced number of admittances to the resuscitation room of our level 1 trauma centre following a traumatic injury, which can be explained by the reduced number of accidents generally occurring at the workplace, in traffic or at outdoor activities. While the number of patients admitted to our institution due to accidental trau- matic injury decreased, the number of patients admitted following an intentional traumatic injury due to a suicide attempt increased. Therefore, the percentage of patients admitted following a suicide attempt during the COVID-19 lockdown was significantly higher compared to the previous 5 years.

The reasons that lead an individual to suicidal behavior are still not fully understood, as the causes are complex and multifactorial [27]. One of the reasons for the significantly higher percentage of suicide-associated admissions to our resuscitation room during the COVID-19 lockdown might be the increased prevalence of depression and anxiety symptoms in this period, compared to previous epidemiological data in Austria [28]. Interestingly, when Pieh et al. performed the same survey they conducted in Austria on a study population in the United Kingdom, they found that the prevalence of severe depressive, anxiety and insomnia symptoms was about three times higher there than in Austria [29]. These findings lead to the hypothesis that other countries might have experienced an even higher increase in the rate of suicide attempts than our Austrian study population.

A level 1 trauma centre is a verified facility that provides the highest level of surgical care to trauma patients and has a full range of specialists and equipment available $24 \mathrm{~h}$ per day. The Medical University of Vienna is the only level 1 Trauma Centre in the greater Vienna region with two resuscitation rooms, where patients can be treated simultaneously. Additionally, it is known that polytrauma patients have a significantly lower mortality when care is obtained at a trauma center [8]. Therefore, it stands to reason that our institution received an influx of major trauma and polytrauma patients during this time period. 
The absolute number of suicide-related polytraumas as well as the proportion of suicide-related polytraumas in comparison to all admissions was higher in our study group than in the groups referring to 2015, 2016, 2018, and 2019. Surprisingly, we detected a spike of suicide-associated polytrauma patients admitted to our institution for the 2-month period in 2017 and further investigated the reasons for this occurrence. During the spring of 2017 another major hospital in Vienna had partially closed its trauma center due to a change in infrastructure [30]. For this reason, data on polytrauma from 2017 are not comparable and were excluded from our analysis. We further studied if a difference in suicide-associated polytrauma admittance could be detected according to the week of admission. Although no suicide-associated polytrauma was treated during the first 2 weeks of the lockdown, no significant difference could be detected; however, this might also be a result of our small sample size. Moreover, we could not find a difference in the mechanism of injury between the study and control groups.

Risk factors associated with higher suicide rates, such as age, gender, substance misuse and mental health concerns, such as depression have been identified [21] and thus at-risk populations defined. It was hypothesized that the number of suicide attempts in the senior population could increase as a result of social isolation during lockdown [26]; however, our data showed no difference in the age distribution of patients who attempted suicide during the lockdown from those in the previous 4 years. Regarding gender, we detected that more males than females attempted suicide in both study and control groups, which is in line with previously published literature [7].

Due to the small number of patients in our study group we could not observe an increase in suicidal behavior isolated for individuals with previous mental health problems, but more generally an increase in suicidality across different groups at risk. Nevertheless, people suffering from psychiatric disorders or mental health conditions tend to be more susceptible to stress than the general population and could therefore experience relapses, new manifestations or substantial worsening of symptoms, such as depression, anxiety, and posttraumatic stress. It follows that all these factors could lead to increased psychological distress and thus lower the inhibition to perform a suicide attempt. Furthermore, many outpatient clinics were closed during the lockdown period and thus regular visits could not take place and the continuance of prescriptions was not always guaranteed, increasing the burden on patients already suffering from mental health symptoms even further [31].

The COVID-19 pandemic has become an amplifier of already existing socioeconomic vulnerabilities and its economic fallout might also lead to further healthcare threats. It is known that unemployed individuals have a higher suicide risk than their employed counterparts and that during spikes of unemployment rates a spike in the number of suicides can also be detected [32-34]. Considering the current global unemployment rates and their projected rise, an increase in suicide attempts should also be expected and prepared for by the mental health community $[35,36]$. Unfortunately, we were unable to assess the current employment status of our study population and can therefore not comment if the economic instability led to the increase of suicidality in our study group. We did analyze the various zip codes to determine the socioeconomic background through the patients' district of residence; however, no geographical cluster could be detected. We also found no association between suicide attempts during the COVID-19 lockdown and belonging to an ethnic minority, even if it has been hypothesized that ethnic minorities, especially migrant populations, such as refugees, migrant workers and asylum seekers could be exponentially more affected by the current pandemic as they are especially vulnerable to the aforementioned dynamics [37].

\section{Strengths and limitations}

Many researchers discussed the possible impact of the COVID-19 lockdown on mental health and suicide attempts; however, to our knowledge this is the first study statistically confirming this hypothesis. By observing the number of suicide-associated admissions to the resuscitation room of our level 1 trauma centre, we are one of the few studies publishing actual data on how many suicide attempts were hospitalized during the lockdown period.

We are aware of the limitations of our study. By the design of our study we had only access to the number and mechanism of injury admitted to the resuscitation room of the level 1 Trauma Centre of the Medical University of Vienna and cannot estimate how many patients received care in other departments or hospitals in the greater Vienna area or Austria in general; however, the absolute number of suicidal acts performed will always be impossible to determine as many individuals do not seek treatment in a hospital or help from mental health professionals after attempting suicide.

\section{Conclusion}

Our findings show that during the lockdown in Austria a significantly higher percentage of patients admitted to the resuscitation room of the level 1 Trauma Centre sustained their injuries due to a suicide attempt by intentional trauma compared to the same 2-month period in the pooled previous 4 years. Therefore, it is crucial to consider mental health and psychological well-being when establishing lockdown policies. Furthermore, healthcare professionals working in trauma centres and first responders should be aware of the needs of patients admitted after attempting suicide during a lockdown. Therefore, consultation of psychi- 
atric and psychological services should be considered from an early point of intervention. We recommend an early interdisciplinary approach.

Author Contribution G.L. Carlin: project development, data collection, manuscript writing; shared first author. J.S. Baumgartner: data collection, manuscript editing; shared first author. T. Moftakhar: data collection, manuscript editing. D. König: manuscript editing. L.L. Negrin: project development, data analysis, manuscript editing. All authors approved the final version of the manuscript.

Funding Open access funding provided by Medical University of Vienna.

\section{Declarations}

Conflict of interest G.L. Carlin, J.S. Baumgartner, T. Moftakhar, D. König and L.L. Negrin declare that they have no competing interests.

Ethical standards For this article no studies with human participants or animals were performed by any of the authors. All studies mentioned were in accordance with the ethical standards indicated in each case. This article describes a retrospective data analysis, which was approved by the local ethics committee (EK no: 1607/2020).

Open Access This article is licensed under a Creative Commons Attribution 4.0 International License, which permits use, sharing, adaptation, distribution and reproduction in any medium or format, as long as you give appropriate credit to the original author(s) and the source, provide a link to the Creative Commons licence, and indicate if changes were made. The images or other third party material in this article are included in the article's Creative Commons licence, unless indicated otherwise in a credit line to the material. If material is not included in the article's Creative Commons licence and your intended use is not permitted by statutory regulation or exceeds the permitted use, you will need to obtain permission directly from the copyright holder. To view a copy of this licence, visit http://creativecommons.org/licenses/by/4.0/.

\section{References}

1. Bikbov B, Perico N, Remuzzi G. Mortality landscape in the global burden of diseases, injuries and risk factors study. EurJ Intern Med. 2014;25:1-5.

2. Chen Y-Y, Chien-Chang Wu K, Yousuf S, Yip PS. Suicide in Asia: opportunities and challenges. Epidemiol Rev. 2012;34:129-44.

3. Curtin SC, Warner M, Hedegaard H. Increase in suicide in the United States, 1999-2014. US Department ofHealth and Human Services, Centers for Disease Control and; 2016.

4. WHO. Global Health Observatory (GHO) data. 2018. https://www.who.int/gho/mental_health/suicide_rates/ en/. Accessed6 July 2020.

5. Olfson M, Blanco C, Wall M, Liu S-M, Saha TD, Pickering RP, et al. National trends in suicide attempts among adults in the United States. JAMA Psychiatry. 2017;74:1095-103.

6. WHO. Preventing suicide: a global imperative. Stylus Publishing; 2014

7. BMSGPK. Suizid und Suizidprävention in Österreich. Wien: Bundesministerium für Soziales, Gesundheit, Pflege und Konsumentenschutz;2020.
8. MacKenzie EJ, Rivara FP, Jurkovich GJ, Nathens AB, Frey KP, Egleston $\mathrm{BL}$, et al. A national evaluation of the effect of trauma-center care on mortality. N Engl J Med. 2006;354:366-78.

9. Akin L, Gözel MG. Understanding dynamics of pandemics. TurkJ Med Sci. 2020;50:515-9.

10. KawohlW, NordtC.COVID-19, unemployment, and suicide. LancetPsychiatry. 2020;7:389-90.

11. Connolly K. Austria reopens small shops and parks as coronavirus lockdown is relaxed. The Guardian. 2020. https://amp.theguardian.com/world/2020/apr/14/ austria-reopens-small-shops-and-parks-as-coronaviruslockdown-is-relaxed. Accessed: 16 May 2020

12. Coccolini F, Perrone G, Chiarugi M, Di Marzo F, Ansaloni L, Scandroglio I, et al. Surgery in COVID-19 patients: operational directives. World J Emerg Surg. 2020;15:25.

13. COVIDSurg Collaborative. Globalguidance for surgical care during the COVID-19 pandemic. Br J Surg. 2020; https:// doi.org/10.1002/bjs.11646.

14. DeSimone B, ChouillardE, Di Saverio S, Pagani L, Sartelli M, Biffl W, et al. Emergency surgery during the COVID-19 pandemic: what you need to know for practice. Ann R Coll Surg Engl. 2020;102:323-32.

15. Gok AFK, Eryilmaz M, Ozmen MM, Alimoglu O, Ertekin C, Kurtoglu MH. Recommendations for trauma and emergencygeneral surgerypractice duringCOVID-19 pandemic. Ulus Travma Acil Cerrahi Derg. 2020;26:335-42.

16. HaddadFS. COVID-19 and orthopaedic and traumasurgery. Bone Joint J.2020;102-B:545-6.

17. Stensland KD, Morgan TM, Moinzadeh A, Lee CT, Briganti A, Catto JWF, et al. Considerations in the triage of urologic surgeries during the COVID-19 pandemic. Eur Urol. 2020;77:663-6.

18. Gunnell D, Appleby L, Arensman E, Hawton K, John A, Kapur N, et al. Suicide risk and prevention during the COVID-19 pandemic. Lancet Psychiatry. 2020. http://www.sciencedirect.com/science/article/ pii/S2215036620301711. https://doi.org/10.1016/S22150366(20)30171-1

19. Santini ZI, Jose PE, Cornwell YE, Koyanagi A, Nielsen L, Hinrichsen C, et al. Social disconnectedness, perceived isolation, and symptoms of depression and anxiety among older Americans (NSHAP): a longitudinal mediation analysis. Lancet Public Health. 2020;5:e62-e70.

20. Weems CF, Carrion V, McCurdy B, Scozzafava MD. Increased risk of suicide due to economic and social impacts of social distancing measures to address the Covid-19 pandemic: a forecast. 2020. https://doi.org/10.13140/RG.2.2.21601. 4592

21. Draper BM. Suicidal behaviour and suicide prevention in later life. Maturitas. 2014;79:179-83.

22. Reger MA, StanleyIH, JoinerTE. Suicidemortality and Coronavirus disease 2019 - a perfect storm? JAMA Psychiatry. 2020; https://doi.org/10.1001/jamapsychiatry.2020.1060.

23. Armitage R, Nellums LB. COVID-19 and the consequences of isolating the elderly. Lancet Public Health. 2020;5:e256.

24. Druss BG. Addressing the COVID-19 pandemic in populations with serious mental illness. JAMA Psychiatry. 2020; https://doi.org/10.1001/jamapsychiatry.2020.0894.

25. Holmes EA, O'Connor RC, Perry VH, Tracey I, Wessely S, ArseneaultL, etal. Multidisciplinary research priorities for the COVID-19 pandemic: a call for action for mental health science. Lancet Psychiatry. 2020. http://www.sciencedirect. com/science/article/pii/S2215036620301681.https://doi. org/10.1016/S2215-0366(20)30168-1

26. Wand APF, Zhong B-L, Chiu HFK, Draper B, De Leo D. Covid-19: the implications for suicide in older adults. 
Int Psychogeriatr. 2020; 1-16. https://doi.org/10.1017/ S1041610220000770

27. O'Connor RC, Nock MK. The psychology of suicidal behaviour. Lancet Psychiatry. 2014;1:73-85.

28. Pieh C, Budimir S, Probst T. The effect of age, gender, income, work, and physical activity on mental health during coronavirus disease (COVID-19) lockdown in Austria. J Psychosom Res. 2020; 110186. https://doi.org/10.1016/j. jpsychores.2020.110186

29. Pieh C, Budimir S, ProbstT. Mental health during COVID-19 Lockdown: a comparison of Austria and the UK-preprint. SSRNJ. 2020; https://doi.org/10.2139/ssrn.3592372

30. APA. Unfallspital Lorenz-Böhler: Schockraum jederzeit einsetzbar. derstandard. 2017. https://www.derstandard. at/story/2000060721790/lorenz-boehler-schockraumjederzeit-einsetzbar. Accessed 6 July 2020.

31. Yao $\mathrm{H}$, Chen J-H, Xu Y-F. Patients with mental health disorders in the COVID-19 epidemic. Lancet Psychiatry. 2020;7:e21.

32. Lundin A, Hemmingsson T. Unemployment and suicide. Lancet. 2009;374:270-1.
33. Milner A, Page A, LaMontagne AD. Long-term unemployment and suicide: a systematic review and meta-analysis. PLoSONE. 2013;8:e51333.

34. Stuckler D, Basu S, Suhrcke M, Coutts A, McKee M. The public health effect of economic crises and alternative policy responses in Europe: an empirical analysis. Lancet. 2009;374:315-23.

35. Deady M, Tan L, Kugenthiran N, Collins D, Christensen H, Harvey S. Unemployment, suicide, and COVID-19: using the evidence to plan for prevention. Med J Aust. 2020; https://doi.org/10.5694/mja2.50715

36. Klomek AB. Suicide prevention during the COVID-19 outbreak. Lancet Psychiatry. 2020;7:390.

37. Ullah A, Nawaz F, Chattoraj D. Locked up under Lockdown the COVID-19 pandemic and the migrant population. SSHO-D-20-00176. 2020.

Publisher's Note Springer Nature remains neutral with regard to jurisdictional claims in published maps and institutional affiliations. 